\title{
Mobilité humaine et changement environnemental : une analyse historique et textuelle de la politique des Nations Unies
}

Human mobility and environmental change: A historical textual analysis of

United Nations' policy

Karen Elizabeth McNamara et Chris Gibson

\section{(2) OpenEdition \\ Journals}

\section{Édition électronique}

URL : http://journals.openedition.org/conflits/18573

DOI : $10.4000 /$ conflits. 18573

ISSN : $1777-5345$

\section{Éditeur :}

CCLS - Centre d'études sur les conflits lilberté et sécurité, L'Harmattan

\section{Édition imprimée}

Date de publication : 30 décembre 2012

Pagination : 43-60

ISBN : 978-2-343-00589-8

ISSN : $1157-996 X$

\section{Référence électronique}

Karen Elizabeth McNamara et Chris Gibson, « Mobilité humaine et changement environnemental : une analyse historique et textuelle de la politique des Nations Unies », Cultures \& Conflits [En ligne], 88 | hiver 2012, mis en ligne le 15 mars 2014, consulté le 30 mars 2021. URL : http://

journals.openedition.org/conflits/18573; DOI : https://doi.org/10.4000/conflits.18573 


\title{
Mobilité humaine et changement environnemental : une analyse historique et textuelle de la politique des Nations Unies ${ }^{1}$
}

\section{Karen Elizabeth McNAMARA, Chris GIBSON}

Karen McNamara est chargée de recherche à l'Ecole de Géographie de l'Université de Queensland en Australie. Ses recherches portent sur les moyens de subsistance durables, le genre, le développement, l'adaptation au changement climatique et les conséquences sociales des changements environnementaux. Elle s'intéresse en particulier aux questions de déplacement forcé de communautés.

Chris Gibson est professeur de géographie bumaine à l'Université de Wollongong, en Australie, où il est également directeur adjoint du Centre australien pour la recherche sur la culture environnementale. Il est l'auteur d'un récent ouvrage intitulé La durabilité des ménages : Défis et dilemmes de la vie quotidienne (Edward Elgar, 2013).

\begin{abstract}
A près les horreurs de la Grande Dépression et de la Seconde Guerre monAdiale, l'Organisation des Nations Unies (ONU) a reçu un mandat très large pour améliorer la paix future et le bien-être de l'humanité. Malgré son travail considérable dans les sphères sociale et économique au cours des soixante dernières années, l'aggravation de la pauvreté, l'injustice et les violations des droits de l'Homme ont été couplées avec la dégradation de l'environnement mondial. Pris ensemble, ces problèmes soulignent les défis consistant à assurer à la fois un environnement global durable et la sécurité de la vie humaine sur terre.
\end{abstract}

Une préoccupation urgente, aujourd'hui, est la protection internationale des personnes qui quittent leur foyer en raison des changements environnementaux. Il n'y a pas de mécanismes officiels et institutionnalisés pour proté-

1. Texte traduit de l'anglais par Marie Georgopoulos. Toutes les traductions des citations anglaises sont du même traducteur. 
ger ces personnes qui se déplacent principalement en raison de tels changements. Les lois internationales ne reconnaissent pas le changement de l'environnement comme un moteur majeur de mobilité, même si certains cadres législatifs nationaux intègrent les déplacements environnementaux forcés ${ }^{2}$. Au cours des quarante dernières années, différentes catégories nouvelles sont apparues, faisant référence aux mouvements de populations dus à la désertification, aux tremblements de terre, aux cyclones, aux accidents chimiques, aux grands barrages et, plus récemment, au changement climatique, à l'élévation du niveau de la mer et à la perte des habitats côtiers. Des expressions telles que « réfugiés environnementaux » et « migrants environnementaux » abondent dans les rapports officiels des Nations Unies ainsi que dans la presse grand public. L'accent est mis en particulier sur les catastrophes naturelles et les changements climatiques ${ }^{3}$.

Plus récemment, de nombreux débats ont émergé au sein des milieux académiques sur l'identification juridique des « réfugiés environnementaux ». La question se pose de savoir dans quelle mesure la reconnaissance du terme " réfugiés environnementaux » permettra une protection des personnes contraintes à se déplacer en raison de changements environnementaux extrêmes et si cela constitue le seul moyen de leur offrir une protection adéquate ${ }^{4}$. Par exemple, Biermann et Boas, en ce qui concerne le changement climatique, considèrent comme positive l'adoption d'un nouveau cadre juridique pour les réfugiés climatiques en vertu de la Convention-cadre des Nations Unies sur les changements climatiques (CCNUCC) ${ }^{5}$. Au contraire, McAdam ${ }^{6}$ se montre sceptique quant à la capacité d'un instrument multilatéral ou d'un traité de résoudre la question du déplacement climatique, en raison de la diversité des mouvements induits, des besoins spécifiques des communautés touchées, du nombre de défis à relever et des difficultés liées à l'adoption et à la mise en ouvre d'un tel texte au niveau international.

Cet article présente un recensement des documents qui constituent la base de la politique des Nations Unies au cours des quarante dernières années et

2. Kälin W., "Conceptualising climate-induced displacement”, in McAdam J. (ed.), Climate Change and Displacement: Multidisciplinary Perspectives, Oxford, Hart Publishing, 2010, pp. 81-104.

3. Buerk R., "Flooded future looms for Bangladesh", BBC News, 12 juillet 2004 ; Branigan T., "If the land gets sick and dies, so will the people", The Guardian, 12 mai 2004 ; Kirby A., "Climate victims 'are refugees", $B B C$ News, 1er octobre 2003 ; Kirby A., "Pacific islanders flee rising seas", $B B C$ News, 9 octobre 2000 ; McGirk T., "Environmental refugees", Time Magazine, 20 mars 2000 ; Kirby A., "West warned on climate refugees", BBC News, 24 janvier 2000.

4. Stal M., Warner K., "The way forward: Researching the environment and migration nexus", UNU-EHS Research Brief, Bonn, UNU-EHS, 2009; Renaud F., Bogardi J., Dun O., Warner K., "Control, adapt or flee: How to face environmental migration", InterSecTions, 5, Bonn, UNU-EHS, 2007 ; Bates D.C., "Environmental refugees? Classifying human migrations caused by environmental change", Population and Environment, 23, 2002, pp. 465-477.

5. Biermann F., Boas I., "Protecting climate refugees: The case for a global protocol", Environment, 50, 2008, pp. 8-16. 
présente des processus de catégorisations mouvantes portant sur des objets eux-mêmes mobiles. Il tente d'examiner, dans une perspective historique, les différentes descriptions textuelles des personnes déplacées du fait des changements environnementaux ainsi que l'utilisation et l'exploitation qui en sont faites par diverses agences des Nations Unies. Ceci est pertinent dans la mesure où la littérature sur le sujet, en plein développement, montre l'importance des points de vue (subjectivation) des populations vulnérables sur les conditions dans lesquelles elles vivent ${ }^{7}$. Ces travaux ont montré par exemple que la catégorie " réfugié environnemental » peut être dévalorisante voire méprisante, et qu'elle est souvent combattue par ceux qu'elle cherche à classifier ${ }^{8}$.

Cet article n'a pas pour vocation de défendre une forme de protection pour les populations vulnérables, ni de faire croire que la protection juridique arrivera aisément à résoudre cet enjeu ${ }^{9}$. Nous nous interrogeons plutôt sur l'absence de protection légale spécifique pour les déplacés environnementaux au sein des structures juridiques des Nations Unies. Selon nous, l'une des explications de cette absence est à la fois historique et sémantique, liée à la manière dont les domaines de connaissances se rapportant aux réfugiés et aux changements environnementaux ont été tenus à l'écart du langage utilisé dans les débats et les documents de politique publique.

Cette analyse retrace donc les différentes façons qui ont été utilisées par les agences des Nations Unies et des agences subsidiaires depuis 1970 pour décrire ceux qui quittent leurs foyers en raison de contraintes environnementales. Grâce à une analyse approfondie de documents d'archives à New York et dans d'autres villes disposant d'agences onusiennes, cet article éclaire ces différentes descriptions textuelles en ciblant six «moments » clés au cours desquels certaines catégories ont été développées, changées ou remises en question. Ces «moments » correspondent notamment à des colloques (tels que les grandes conférences des Nations Unies), à la publication de textes officiels ou de rapports de recherche émis par diverses agences onusiennes ou à des projets de financement (complet ou partiel) de ces initiatives. Ces « moments » et les textes qui y sont associés n'ont pas été choisis parce qu'ils avaient en euxmêmes une influence importante sur le débat, mais parce qu'ils mettent en évidence comment des catégories de population particulières ont été créées de

6. McAdam J., "Swimming against the tide: Why a climate change displacement treaty is not the answer”, International Journal of Refugee Law, 23, 2011, pp. 1-26.

7. Farbotko C., Lazrus, H., "The first climate refugees? Contesting global narratives of climate change in Tuvalu”, Global Environmental Change, 22, 2012, pp. 382-390.

8. Kelman I., "Hearing local voices from Small Island Developing States for climate change", Local Environment, 15, 2010, pp. 605-619 ; McAdam J., Loughry M., "We aren't refugees”, Inside Story, 29 juin 2009 ; McNamara K.E., Gibson C., "'We do not want to leave our land': Pacific ambassadors at the United Nations resist the category of 'climate refugees'”, Geoforum, 40, 2009, pp. 475-483 ; Masquelier A., “Why Katrina’s victims aren't refugees: Musings on a 'dirty' word”, American Anthropologist, 108, 2006, pp. 735-743.

9. McAdam J., 2011, op. cit. 
différentes façons pour générer certains types de résultats dans la mise en place de politiques publiques.

Nous tenterons d'explorer la façon dont ces catégories ont été créées en relation avec des conceptualisations assez particulières des notions de «société » et de «nature », et des perceptions différentes quant aux déclencheurs, de courte ou de longue durée, des déplacements de population. Ces descriptions textuelles de la mobilité humaine liée aux changements environnementaux s'appuient inévitablement sur, et en retour forgent, la façon dont sont perçus les processus naturels, les événements et les catastrophes ${ }^{10}$. Dans chacun des «moments » évoqués ci-dessus, des institutions et des agents particuliers établissent des interprétations et des relations de causalité entre les processus environnementaux et des événements en relation avec les populations humaines. "La parole sur la nature 11 ", qui traite des catastrophes environnementales, influence fortement la façon de percevoir ces populations humaines, et donc le contexte plus large des politiques publiques mises en œuvre. Cette étude se rapproche ainsi de la littérature sur les questions des relations entre humains et non-humains 12 et de la construction sociale des catastrophes naturelles ${ }^{13}$.

\section{Le cadre de recherche}

Afin d'accéder aux archives des Nations Unies, un des auteurs a pris un poste de stagiaire auprès du Global Policy Forum, basé à New York, une organisation non gouvernementale qui évalue le processus décisionnel et l'application du principe de responsabilité de l'ONU. Ce stage a permis à ce chercheur d'accéder à l'institution et d'obtenir des entretiens avec des diplomates et ambassadeurs. L'analyse de cette dernière source d'information dépassant le cadre de cet article, elle est examinée ailleurs ${ }^{14}$. Au cours d'une période de six mois, des archives, des publications et des débats ont été examinés, notamment aux agences des Nations Unies situées à New York, Washington, Londres, Genève et Bangkok. À partir de plus d'un millier de documents publics et confidentiels qui ont été trouvés dans les archives, cet article se focalise sur six principaux «moments » où les changements dans la définition de la mobilité humaine liée aux crises environnementales étaient les plus perceptibles.

10. Tastree N., Nature, Londres, Routledge, 2005.

11. Ibid.

12. Castree N., Nash C., "Posthuman geographies", Social and Cultural Geography, 7, 2006, pp. 501-504 ; Head L., Muir P., "Suburban life and the boundaries of nature: Resilience and rupture in Australian backyard gardens", Transactions of the Institute of British Geographers, 31, 2006, pp. 505-524 ; Castree N., 2005, op. cit. ; Demeritt D., "Being constructive about nature”, in Castree N., Braun B. (eds.), Social Nature: Theory, Practice, and Politics, Oxford, Wiley-Blackwell, 2001, pp. 22-40.

13. Pelling M., “Natural disasters?”, in Castree N., Braun B. (eds.), op. cit., pp. 170-181.

14. McNamara K. E., Gibson C., 2009, op. cit. ; McNamara K.E., "Conceptualizing discourses on environmental refugees at the United Nations”, Population and Environment: A Journal of Interdisciplinary Studies, 29, 2007, pp. 12-24. 
La principale période concernée par la recherche des archives va de 1970 à 2010. L'année 1970 a été choisie pour commencer l'analyse détaillée de cette généalogie car elle coïncide avec le début de l'ère environnementale contemporaine ${ }^{15}$. Pendant la période 1970-2010, les associations environnementales ont prospéré et il y a eu une sensibilisation sociale accrue quant à la possibilité d'une crise écologique globale ${ }^{16}$. Ce mouvement environnemental grandissant a suscité la première Conférence des Nations Unies sur l'environnement, qui s'est tenue à Stockholm en 1972. La conférence a abouti à un plan d'actions composé de 109 recommandations, ainsi qu'à la mise en place du Programme des Nations Unies pour l'environnement (PNUE) ${ }^{17}$.

\section{«Moment $1 »$ : Les premiers écrits sur les réfugiés écologiques}

En 1974, Lester Brown a fondé le Worldwatch Institute, une ONG basée à Washington. Le PNUE y était impliqué et a parrainé l'un des projets initiaux de l'Institut en 1975, qui examinait l'impact des systèmes de production intensive d'aliments sur l'environnement, tels que la surpêche, la culture intensive, la production de bois de chauffage et le surpâturage. Les publications issues de ce projet comprenaient une évaluation des liens entre la migration accrue (interne et internationale) et les problèmes environnementaux (déforestation, avancée du désert, épuisement des sols et érosion) ${ }^{18}$.

Le principal argument avancé dans ces publications est que, pendant les années 1970, les industries agricoles ne pouvaient pas ne pas soutenir la croissance de la population, ni accompagner la croissance ultérieure de la population active. Ces arguments s'appuyaient sur des préoccupations malthusiennes selon lesquelles la main-d'œuvre ne pourrait pas maintenir un rythme soutenu de croissance de la population en grande partie en raison des systèmes de propriété foncière inégaux et de la dégradation anthropique de l'environnement ${ }^{19}$. La conséquence de cela devait être des migrations à grande échelle, principalement en Asie et en Afrique, dans les zones urbaines, les forêts tropicales, les collines, les pâturages, les zones à risque de catastrophes naturelles, et les pays voisins. Ces publications ont établi des liens entre la dégradation de l'environnement et les déplacements de population, en utilisant la terminologie présentée ci-après.

15. Young J., Sustaining the Earth: The Past, Present and Future of the Green Revolution, Sydney, New South Wales University Press, 1991.

16. Beder S., The Nature of Sustainable Development, Newham, Scribe, 1996.

17. Aplin G., Mitchell P., Cleugh H., Pitman A., Rich D., Global Environmental Crises: An Australian Perspective, Melbourne, Oxford University Press, 1995.

18. Brown L., McGrath P., Stokes B., "Twenty-two dimensions of the population problem", Worldwatch Paper, 5, Washington, Worldwatch Institute, 1976 ; Eckholm E., Losing Ground: Environmental Stress and World Food Prospects, New York, Oxford Geography Series, 1976 ; Brown L., "The politics and responsibility of the North American Breadbasket", Worldwatch Paper, 2, Washington, Worldwatch Institute, 1975 ; Eckholm E., "The other energy crisis: Fuelwood", Worldwatch Paper, 1, Washington, Worldwatch Institute, 1975.

19. Malthus T., An Essay on the Principle of Population, 1ère edition, Penguin Classics, 1798. 
Comme les populations humaines et animales fuient avant que le désert ne s'étende, ces réfugiés écologiques créent une pression encore plus grande sur les nouvelles zones limitrophes, exacerbent les processus de dégradation des sols, et déclenchent un cercle vicieux de surpopulation et de surpâturage dans les zones successivement occupées 20.

Brown et ses coauteurs donnent des exemples précis, comme l'expansion du Sahel vers le sud en Afrique et le Dust Bowl aux États-Unis 21. Dans ces descriptions, les réfugiés écologiques ont été définis comme les auteurs de la dégradation de l'environnement en raison de l'emploi de pratiques telles que le surpâturage, la production agricole intensive et la surpêche. Par conséquent, les auteurs de ces publications (et en particulier Brown, le fondateur de l'institut), étaient parmi les premiers à populariser le lien entre dégradation de l'environnement et déplacements de population, et à utiliser le terme « réfugiés écologiques » pour décrire un tel phénomène. Ces constructions et concepts ont encore été mûris dans les décennies suivantes par des études menées ou financées par les agences des Nations Unies.

\section{«Moment 2 »: L’émergence de la catégorie « réfugié environnemental » à l'Organisation des Nations Unies}

Dix ans après les principales publications de l'Institut Worldwatch, le PNUE publie un rapport sur les « réfugiés environnementaux ». Bien qu’il soit difficile de comprendre pourquoi un écart de dix ans sépare ces deux moments du discours politique, les raisons pour lesquelles le PNUE a demandé au professeur El-Hinnawi de mener des recherches sur le phénomène des « réfugiés environnementaux » paraissent plus évidentes du fait de l'influence de la deuxième vague de l'environnementalisme ${ }^{22}$, et des chiffres officiels révélant une augmentation du nombre de personnes déplacées à la suite de changements environnementaux. Selon Tolba, à l'époque directeur exécutif du PNUE, les « réfugiés environnementaux » comprenaient les « millions de personnes fuyant les sécheresses d'Afrique du Nord, les victimes de Bhopal et les milliers de sans-abri victimes du séisme de Mexico ${ }^{23}$ ». ElHinnawi a siégé dans différents groupes d'experts des Nations Unies dans les années 1990 mais il reste plus connu à l'ONU pour son article de 1985, où il décrit trois types de "réfugiés environnementaux».

Le premier type comprend ceux « temporairement déplacés en raison d'un stress environnemental 24 ", comme les inondations, les cyclones tropicaux, les sécheresses, les tremblements de terre. Les pays les plus pauvres caractérisés

20. Brown L. et al., 1976, op. cit., p. 39.

21. Ibid.

22. Young J., 1991, op. cit.

23. Tolba M., "Forward", in El-Hinnawi E., Environmental Refugees, Nairobi, UNEP, 1985, p. i.

24. El-Hinnawi E., Environmental Refugees, Nairobi, UNEP, 1985, p. 4. 
par des processus sévères de la dégradation des sols ont été plus durement touchés par des catastrophes naturelles : « les gens peuvent changer leur environnement en le rendant plus sensible à certains déclencheurs de catastrophes, tels que les inondations et la sécheresse 25 ». El-Hinnawi dépeint ceux qui sont touchés comme des victimes de perturbations naturelles mais aussi comme des facteurs d'aggravation de l'ampleur des catastrophes. Ainsi les « réfugiés environnementaux » sont catalogués comme des victimes temporaires mais aussi comme des causes de la sévérité des catastrophes.

Le deuxième type de "réfugiés environnementaux 》 comprend ceux « définitivement déplacés et réinstallés dans une zone nouvelle 26 » suite à des interventions anthropiques telles que la construction de barrages. Ici, les gens ont été forcés «d'abandonner leurs maisons, leurs champs et leurs biens 27 » dans leur pays d'origine. Avec une mauvaise planification, des budgets insuffisants et des transferts de technologie insuffisants, les «bénéficiaires directs des projets étaient souvent les élites instruites, au pouvoir, tandis que les coûts sociaux directs étaient supportés par la population rurale pauvre $28 »$. ElHinnawi considère ces « réfugiés environnementaux » comme des victimes car ils ne sont pas responsables des changements environnementaux qui leurs sont imposés.

Le troisième et dernier type comprend ceux qui sont touchés par des ressources dégradées à cause de processus de déforestation et de désertification et qui ne sont plus en mesure de subvenir aux besoins des populations humaines. El-Hinnawi soutient que :

\begin{abstract}
«Une grande partie du blâme pour la destruction des forêts tropicales est à la charge de cultivateurs itinérants, ceux qui pratiquent la culture sur brûlis dans une clairière de la forêt, cultivée pendant quelques années jusqu'à ce que la fertilité des sols s'épuise, et qui se déplacent ensuite pour dégager un nouveau terrain 29 ».
\end{abstract}

Beaucoup d'exemples donnés par El-Hinnawi sont tirés de communautés rurales de pays pauvres, où les préoccupations de gains à court terme et de simple survie l'emportaient le plus souvent sur celles de durabilité et de préservation à long terme de l'environnement. Dans son analyse, il discute de la désertification en Afrique sub-saharienne et en Asie du Sud : « la désertification est causée presque entièrement par une mauvaise utilisation humaine de l'environnement 30 ». Il a ainsi soutenu que l'agriculture non durable et les 
pratiques de défrichage pratiquées par les « réfugiés environnementaux » euxmêmes ont déclenché et exacerbé des processus tels que la saturation en eau, l'épuisement des sols, leur érosion et leur salinisation. De cette façon, les " réfugiés environnementaux » ont été définis par El-Hinnawi comme des causes du changement environnemental - leur groupe mettant en ouvre des pratiques préjudiciables à la préservation des ressources localement disponibles - ce qui induit finalement un déplacement permanent de ses membres.

\section{« Moment 3 » : Le début des années 1990 marque la multiplication des conférences onusiennes et un intérêt grandissant sur la question}

En 1992, une conférence internationale sur les migrations et l'environnement se tient quelques mois avant la Conférence des Nations Unies sur l'environnement et le développement (CNUED). Cette conférence ne doit pas être considérée comme un élément séparé du discours politique : elle donne priorité à la production de recommandations sur la façon d'aborder le problème lors de la CNUED, considérée comme une avancée majeur des années 1990. Deux recommandations ont été formulées lors de cette conférence: [a] la CNUED doit s'attaquer aux causes profondes des déplacements environnementaux, [b] aux besoins des victimes. Pour y parvenir, la CNUED est invitée à élaborer un cadre pour l'attribution des responsabilités au sein et entre les États, entre les organisations régionales et entre les organismes internationaux. Malgré l'envergure et l'ampleur du programme de la CNUED, celui-ci n'a pas été à la hauteur de cet objectif. Seules quelques références marginales ont porté sur la mobilité humaine en relation avec les changements environnementaux, et surtout sur la désertification (voir ci-dessous).

La CNUED qui avait comme objectif de proposer des mécanismes réalistes pour garantir un développement écologiquement durable, s'est donc tenue à Rio de Janeiro en 1992. Pour atteindre cet objectif, la CNUED a approuvé et adopté deux principaux documents. Le premier est la Déclaration de Rio, sur la base de 27 principes devant régir le comportement économique et environnemental des nations et des individus ${ }^{31}$. Le second est l'Agenda 21, un plan d'action mondial sur le développement durable pour les gouvernements et les $\mathrm{ONG}^{32}$.

L'Agenda 21 reconnaît que l'état de l'environnement naturel est interconnecté aux activités humaines de plusieurs façons. Il fait pourtant peu référence à la notion de "réfugiés environnementaux », surtout dans le cadre des discussions sur la sécheresse et la désertification. L’Agenda 21 déclare que la « désertification est le résultat de divers facteurs, parmi lesquels les variations climatiques et les activités humaines 33 ». La lutte contre la désertification est l'une

31. ONU, Déclaration de Rio, New York, ONU, 1992.

32. ONU, Agenda 21, New York, ONU, 1992.

33. Ibid., p. 12.E. 
des six recommandations prioritaires figurant dans Action 21, dans lequel un appel est lancé pour l'élaboration de plans de préparation à la sécheresse et de secours en cas de sécheresse, y compris des systèmes d'entraide pour les zones menacées : "préparation à la sécheresse globale, programmes d'allégement, y compris l'auto-assistance, pour zones sujettes aux sécheresses $34 »$. Ce qu'il est important d'observer ici, c'est la notion d'entraide. Elle reconnait que les personnes touchées ont des capacités d'adaptation qui leur sont propres. Or, cette notion peut être aussi interprétée négativement et signifier que ceux qui quittent leur foyer doivent s'adapter en supportant le fardeau du changement induit par la sécheresse et la désertification. Ceci a introduit une nouveauté dans le représentation de ceux qui sont touchés par les changements environnementaux : ils sont devenus des sujets adaptables. L'entraide a été recommandée sans prise en considération des causes à l'origine de ces changements qui peuvent être liées à la nature, aux activités humaines ou à la pauvreté et qui avaient déjà inspiré les représentations précédentes.

L'Institut de la population ${ }^{35}$ a également élaboré un programme pour la Conférence des Nations Unies sur la population et le développement, tenue au Caire en septembre 1994. Or, aucune des recommandations de l'Institut n'a été incluse dans le Programme d'action de la Conférence, et seulement une maigre référence reconnaissait que : « les déséquilibres économiques internationaux, la pauvreté et la dégradation de l'environnement [...] sont autant de facteurs qui influent sur les migrations internationales $36 »$. Ici, le Programme d'action a minoré l'importance des problèmes environnementaux, présentés comme une simple composante d'une série de raisons influençant le choix de quelqu'un qui décide de migrer.

\section{«Moment 4 »: Au milieu des années 1990, apparaît la plus grande étude sur le sujet suivie d'une réponse de l'ONU}

Un an après la Conférence des Nations Unies sur la population et le développement, l'Institut sur le Climat, une ONG basée à Washington, a commandé à Norman Myers, professeur en sciences environnementales, un travail de recherche sur la question des réfugiés environnementaux. Ce projet a donné lieu à un rapport de 214 pages sur les « réfugiés environnementaux 37 ", qui comprenait une série de données sur les chiffres et l'ampleur du problème ${ }^{38}$. Myers a, depuis, révisé ces chiffres à plusieurs reprises. Plus récem-

34. Ibid., p. 12.E.

35. Institut de la Population, Desperate Departures: The Flight of Environmental Refugees, Washington, Institut de la Population, 1992.

36. ONU, Conférence internationale sur la population et le développement, New York, ONU, 1994, article 10.1 .

37. Ce projet a été cofinancé par le FNUAP et le PNUE, le Overseas Development Administration du Royaume-Uni, le Fonds Moriah, le gouvernement des États-Unis, la Fondation Rockefeller et l'Agence suédoise pour le développement international.

38. Myers N., Environmental Exodus: An Emergent Crisis in the Global Arena, Washington, Climate Institute, 1995. 
ment, Stern s'est basé sur les estimations de Myers annonçant qu'il y aura 200 millions de personnes déplacées de façon permanente d'ici à 2050 39. Dans son rapport, Myers identifiait quatre principaux déclencheurs de déplacement, attribuait des responsabilités quant à l'apparition de ces flux et proposait deux constructions identitaires des « réfugiés environnementaux »- les victimes et les victimes réinstallées à l'intérieur du pays. Le premier déclencheur consistait en des changements environnementaux graduels, y compris la dégradation des sols et le changement climatique. Myers attribuait le processus de dégradation des sols à un déficit de terres cultivables, aux pratiques agricoles inadaptées, au manque d'infrastructures rurales pour l'agriculture, à la croissance de la population et à la pauvreté. Ensemble, ces facteurs avaient une incidence sur les moyens «de subsistance d'un grand nombre de personnes, en particulier pour les membres des tribus et autres populations indigènes 40 ». C'était la première fois que les "réfugiés environnementaux » déplacés en raison de la dégradation des sols ont été considérés comme des victimes. Dans le cas du changement climatique, Myers soutient que les impacts du changement climatique et l'exode des populations qu'il génère sont une conséquence directe de l'augmentation des émissions de gaz à effet de serre attribuée aux pays riches. Il définit les actions de ces gouvernements comme des agressions et les « réfugiés environnementaux » de ces actions comme des victimes impuissantes situées à l'extérieur des pays émetteurs. Enfin, il fait valoir que les personnes touchées ne sont pas responsables du processus de changement; la faute doit plutôt être recherchée dans la dépendance des autres pays à l'usage des combustibles fossiles.

Les catastrophes naturelles de grande ampleur sont identifiées par Myers comme le second déclencheur de déplacements des populations. Ces événements ont été «souvent aggravés par les pressions de la population [...] ou la pauvreté ", qui ont rendu de nombreuses communautés vulnérables et dépourvues de moyens nécessaires pour éviter le désastre. Myers exprime de la considération pour les populations qui se trouvent dans une telle situation dans la mesure où, en dépit de la composante humaine des catastrophes naturelles, il ne qualifie pas ceux qui en sont touchés de facteurs aggravant ou contribuant à de tels changements. Au contraire, il souligne que ces « réfugiés environnementaux » sont des victimes de catastrophes naturelles, de la pauvreté généralisée et des pressions démographiques. Son raisonnement, consistant à construire l'identité des " réfugiés environnementaux » en tant que victimes, l'amène à affirmer que ce sont « souvent les personnes les plus pauvres qui sont les plus exposées au risque - et ils sont ceux qui, en vertu de leur situation d'appauvrissement, ne peuvent pas faire grand chose pour se protéger 41 ». Les deux autres déclencheurs qu'il propose sont les accidents environnementaux et les crises environnementales produites par la construction d'in-

39. Stern N., Stern Review on the Economics of Climate Change, Londres, HM Treasury, 2006.

40. Ibid., p. 37.

41. Ibid., p. 25. 
frastructures. Comme dans les précédents "moments ", les personnes touchées sont considérées comme des victimes réinstallées à l'intérieur de leur pays, ceci en raison de la participation directe ou indirecte des gouvernements de ces pays dans l'avènement de tels changements dans l'environnement.

En 1996, le Haut Commissariat aux Réfugiés (HCR) et l'Organisation Internationale pour les Migrations (OIM) organisent, à Genève, un colloque international intitulé « Les déplacements de populations induits par l'environnement et les impacts environnementaux résultant de migrations massives 42 ». L'ouvrage publié à la suite du colloque refuse d'utiliser le terme de "réfugiés environnementaux " afin "d'éviter toute confusion avec la définition juridique des réfugiés 43 » figurant dans la Convention de 1951 ; le terme de "personnes déplacées pour des raisons écologiques » a été préféré. Dans ce cas, les réfugiés de l'environnement ont été différenciés des réfugiés légaux. En outre, les auteurs qualifient les personnes déplacées suite à des modifications environnementales de « migrants». Ils soutiennent que le déplacement massif de personnes - des migrants - en raison d'une dégradation environnementale causait des problèmes au pays d'accueil car les arrivants se trouvaient en situation de concurrence avec les populations locales pour l'exploitation des ressources et l'accès à l'emploi. Ils considèrent ainsi que « dans certains cas, les migrants peuvent fragiliser le tissu social et économique des zones dans lesquelles ils se réinstallent 44 ». Ici, l'accent est mis sur les problèmes qui émergent aux endroits où les gens se sont réinstallés, plutôt que sur la souffrance et la détresse à l'origine des déplacements.

Cette publication décrit cinq grandes catégories de mobilité humaine suite à des changements environnementaux. La première catégorie inclut des situations survenues subitement, causées par des catastrophes naturelles ; cependant, il est reconnu que « la distinction entre les causes naturelles et anthropiques (sic) peut être incertaine 45 ». Ici, les déplacés sont définis comme des "victimes temporaires » et comme des «facteurs aggravants ». La deuxième catégorie inclut la contamination par des déchets toxiques dangereux qui créent également des déplacements massifs de populations. Les auteurs présentent les personnes déplacées par une contamination chimique comme victimes de changements environnementaux survenus au sein des frontières et pour lesquels ils ne sont pas responsables - des « victimes réinstallées à l'intérieur » de leur pays. Ces deux définitions ont donc persisté, et n'ont que très peu changé tout au long des divers «moments » du discours de politique publique.

42. Le Colloque a également reçu un financement supplémentaire du FNUAP, ainsi que des gouvernements du Canada, de l'Allemagne, des Pays-Bas, de la Norvège, de la Suède et de la Suisse.

43. UNHCR, IOM, RPG, Environmentally-induced Population Displacements and Environmental Impacts Resulting from Mass Migrations, Genève, UNHCR, IOM and RPG, 1996, p.10.

44. Ibid., p. 15.

45. Ibid., p. 16. 
Les trois catégories suivantes développées dans l'ouvrage du HCR sont définies par rapport à des changements environnementaux lents (grands barrages, dégradation des sols et changement climatique). Il y est considéré que les personnes déplacées par la construction de grands barrages sont des victimes de dommages environnementaux dont ils ne sont pas responsables. Le HCR ne fait ici aucune mention d cees déplacements transfrontaliers, conséquences de ces développements et dont on peut supposer que les personnes déplacées ont été définies, à l'image des catégories précédentes, comme des victimes réinstallées à l'intérieur de leur pays. La dégradation des sols et le changement climatique sont considérés comme des problèmes irréversibles, mais les auteurs ne font aucune mention directe des causes de ces changements autres que des affirmations générales sur le rôle des activités humaines et des processus naturels. Toutefois, les recommandations fournies ont influencé la façon de percevoir ces personnes déplacées. La principale recommandation consiste à dire que les populations touchées par une évolution lente de l'environnement doivent faire en sorte de s'adapter à un tel changement au travers des campagnes d'éducation sur l'utilisation durable des ressources et par le renforcement de leurs capacités techniques. Les personnes touchées par la dégradation des sols et le changement climatique ont donc été définies comme adaptables.

Ce qui parait évident à travers les «moments » relatés jusqu'ici, c'est que, durant les années 1980 et 1990, le débat autour de ces enjeux était particulièrement important. Cependant, il a perdu en vigueur à la suite du Colloque international de 1996 organisé par le HCR et l'OIM. Malgré d'importantes recherches retrouvées dans les archives de l'Organisation des Nations Unies, dans cinq grandes villes du monde, aucun texte important sur la mobilité humaine et le changement de l'environnement n'a été trouvé depuis cette date et jusqu'à la publication par le HCR d'un rapport de recherche sur les « réfugiés environnementaux » en 2001 (voir ci-dessous). Le colloque de 1996 intervient peu de temps après l'étude sur les « réfugiés environnementaux » de Myers en 1995. Le rapport détaillé de Myers, mentionné précédemment, recommandait un élargissement nécessaire du régime international sur les réfugiés pour y inclure les « réfugiés environnementaux ». Myers faisait valoir qu'une conférence internationale des Nations Unies serait le moyen le plus efficace pour traiter la question de l'acceptation officielle de la catégorie des " réfugiés environnementaux » et il avait présenté ses conclusions au Secrétariat des Nations Unies en 1995 46. Le colloque de 1996 a été l'occasion de développer des mécanismes internationaux visant à reconnaitre officiellement et à protéger les personnes déplacées en raison de contraintes environnementales. Pourtant, cet effort d'action publique a été éphémère; un silence de cinq ans a suivi.

46. Myers N., 1995, op. cit. 


\section{«Moment 5 » : Le tournant du siècle voit la question contestée}

En 2001, après une période de cinq ans, un document de travail du HCR rédigé par l'universitaire et géographe Richard Black est publié, intitulé «Réfugiés de l'environnement : mythe ou réalité ?». Richard Black a longtemps écrit sur les liens entre réfugiés, environnement et développement ${ }^{47}$. En 1998, il a débattu de l'utilisation de l'expression "réfugiés environnementaux », affirmant qu'elle était incorrecte et trompeuse et a soutenu que parmi les preuves existantes, il y avait « étonnamment peu de preuves scientifiques » pour affirmer que le monde se « remplit de réfugiés de l'environnement 48 ». Dans les cas où des projets de recherche ont fourni des preuves de l'existence de " réfugiés environnementaux », Black fait valoir qu'ils comportaient des "lacunes méthodologiques 49 », car la preuve fournie était souvent dissociée des contextes économiques, politiques et sociaux qui pourraient être aussi des causes significatives de déplacement. En outre, Black a souligné l'inexactitude et, dans certains cas, l'absence de statistiques dans la littérature sur les « réfugiés environnementaux », en particulier les estimations faites par Myers et Westing ${ }^{50}$. Selon Black, cette inexactitude était le résultat de statistiques fortement tributaires d'une définition préalable formelle des « réfugiés environnementaux ». Pour lui, le processus d'élaboration d'une définition de « réfugiés environnementaux » serait impossible et inutile, étant donné l'imbrication de multiples causes déclenchant des déplacements de population.

Bien que la publication de Black en 2001 ne représente pas la position officielle du HCR, elle nous montre comment les savoirs d'experts peuvent être créés et appliqués et comment ils peuvent influencer les orientations politiques des agences des Nations Unies, comme le HCR, sur des questions particulières. En 2001, Black soutient donc que le concept de « réfugiés environnementaux » est un mythe, surtout parce que la fuite des réfugiés a souvent plusieurs causes. Par conséquent, isoler les facteurs environnementaux et les présenter comme étant l'unique cause des flux de réfugiés, c'est, pour Black, simplifier la question en évacuant les interactions complexes qui sont à l'origine des déplacements de populations :

47. Black R., “Environmental refugees: Myth or reality?”, UNHCR Working Paper, 34, Genève, UNHCR, 2001 ; Black R., Refugees, Environment and Development, Londres, Longman, 1998 ; Black R., "Environmental change in refugee affected areas of the Third World: The role of policy and research”, Disasters, 18, 1994, pp. 107-115; Black R., Robinson V. (eds.), Geography and Refugees: Patterns and Processes of Change, Londres, Belhaven, 1993.

48. Black R., 1998, op. cit., p. 23.

49. Ibid., p. 23.

50. Myers N., 1995, op. cit. ; Myers N., "Eco-refugees: A crisis in the making”, People and the Planet, 3, 1994, pp. 6-9; Myers N., "Environmental refugees in a globally warmed world", Bioscience, 43, 1993, pp. 752-761 ; Westing A.F., "Environmental refugees: A growing category of displaced persons”, Environmental Conservation, 19, 1992, pp. 201-207 ; Myers N., "Population, environment and conflict", Environmental Conservation, 14, 1987, pp. 15-22. 
«Bien que la dégradation de l'environnement et les catastrophes puissent être des facteurs importants dans la décision de migrer, et constituent en soi des enjeux de préoccupation, leur conceptualisation comme cause principale des déplacements forcés est incohérente et aléatoire intellectuellement, et en pratique inutile ${ }^{51}$. »

Selon cette conceptualisation, les facteurs environnementaux n'agissent pas de manière isolée, mais sont plutôt liés à divers autres facteurs économiques, culturels et politiques en tant que causes de "migrations » ou de "déplacements ». Black examine onze incidents importants, générateurs de réfugiés au cours des années 1990 ; beaucoup portaient sur le contrôle de ressources limitées, en particulier sur les réserves de pétrole au Soudan, en Azerbaïjan et au Kazakhstan. Dans ces différents cas, les facteurs environnementaux ont contribué à des flux de réfugiés. Cependant, d'après Black, ces facteurs étaient des causes de déplacement beaucoup moins importantes que les luttes de pouvoir, la guerre civile et les conflits ethniques, en particulier dans la région des Grands Lacs, en Somalie et en Sierra Leone. Il conclut que, compte tenu du manque de preuves empiriques, la présence de liens solides entre changements environnementaux et mobilité humaine reste à prouver, et donc, qu'en tant que telle, cette catégorie de personnes est considérée comme non légitime.

La proposition de Black consiste à dire que les déclencheurs environnementaux ne sont pas une motivation unique pour les personnes qui cherchent refuge ailleurs. Si le déplacement des gens résulte d'un mélange complexe de raisons, cela ne doit pas pour autant signifier que le changement environnemental n'est pas un facteur important parmi d'autres. L'effet direct de cet argument (le besoin d'identifier clairement les déclencheurs de déplacements) a été un ralentissement dans la prise de mesures au sein de l'arène politique internationale.

\section{«Moment 6 » : Les travaux préparatoires de la Conférence des Parties à la Convention à Copenhague}

À l'approche de la réunion annuelle de la Conférence des parties à Copenhague (COP 15, Convention-cadre des Nations Unies sur les changements climatiques) en décembre 2009 52, une suractivité autour de la question de la mobilité humaine et du changement environnemental a eu lieu. Les rapports et les déclarations sur la COP 15 montrent que cette réunion a été considérée comme un moment critique au sein de la communauté internationale pour s'entendre sur la voie à suivre en matière de lutte contre le changement

51. Black R., 2001, op. cit., p. 1.

52. Étant donné que la recherche d'archives a eu lieu en grande partie en 2005, les conférences et les rapports suivants ont été identifiés à partir d'une recherche sur Internet (à l'aide de Google). 
climatique. Cette voie prônait la diminution des émissions de gaz à effet de serre à des niveaux sûrs, la protection des générations futures, et la réduction du nombre de personnes obligées de quitter leurs lieux habituels de vie.

Pendant cette période, le débat sur la question du déplacement environnemental s'est déporté vers celle du déplacement dû au changement climatique ${ }^{53}$. Le projet EACH-FOR 54 fut une exception à cette évolution dans le sens où il examinait tous les types de processus de changements environnementaux comme déclencheurs, principaux ou secondaires, de déplacements des populations 55. Même si le financement de ce projet était assuré par l'Union Européenne, sa réalisation était assurée par l'Institut Universitaire des Nations Unies pour l'Environnement et la Sécurité Humaine (UNU-EHS). Cette première enquête globale sur la migration environnementale dans 23 pays a permis d'identifier des facteurs directs et indirects de migrations forcées, d'élaborer une série de scénarios de " migrations forcées », et de réaliser des études de cas sur les liens entre dégradation de l'environnement et migration. Ses auteurs constatent qu'il existe des facteurs à la fois sociaux, politiques et économiques qui peuvent influer sur la mobilité humaine, parallèlement au changement environnemental. Dans le prolongement de cette complexité, ils ont également soutenu que la dégradation de l'environnement est le résultat de la détérioration des structures sociales, économiques et politiques. En réponse à cette complexité, ils ont élaboré un cadre décisionnel permettant de classer les personnes déplacées du fait d'une détérioration de l'environnement 56 .

À la même époque, l'UNU-EHS, avec l'ONG CARE, a également publié un rapport sur le changement climatique et le déplacement ${ }^{57}$. Ce rapport a été publié avant une réunion entre responsables gouvernementaux et autres parties prenantes en Allemagne, en juin 2009, pour décider des questions à inclure dans l'accord mondial sur le changement climatique auquel, espéraient-ils, la COP 15 allait aboutir. Financé par le HCR et la Banque mondiale, ce rapport a été publié pour aider les décideurs à mieux comprendre les enjeux relatifs aux migrations climatiques et aux déplacements comme modalités d'adapta-

53. Piguet E., "Climate change and forced migration", New Issues in Refugee Research (Research Paper no 153), Genève, UNHCR, 2008 ; Brown O., "Climate change and forced migration: Observations, projections and implications", Thematic Paper for the Human Development Report 2007/2008, Genève and New York, UNDP, 2007.

54. EACH-FOR, "Preliminary findings from the EACH-FOR project on environmentally induced migration", Évolution de l'environnement et scénarios de migration forcée, Bonn, UNU-EHS, 2008.

55. Renaud F., Dun O., Warner K., Bogardi, J., “A decision framework for environmentally induced migration”, International Migration, 49, 2011, pp. 5-29 ; Dun O., Gemenne F., "Defining environmental migration", Forced Migration Review, 31, 2008, pp. 10-11; Vlassopoulou C. A. (ed.), «Exodes écologiques : L’environnement source de migrations », Revue en ligne Asylon(s), novembre 2008; Renaud F. et al., 2007, op. cit.

56. Renaud F. et al., 2011, op. cit.

57. CARE, In Search of Shelter: Mapping the Effects of Climate Change on Human Migration and Displacement, USA, CARE, 2009. 
tion. Tout au long de ce rapport, la migration et le déplacement sont des termes utilisés pour décrire ceux qui cherchent refuge dans leur propre pays ou en franchissant les frontières, pour répondre aux crises et aux changements environnementaux. Bien que ce rapport souligne la nécessité pour les personnes déplacées par le changement climatique d'être reconnues, il utilise de façon interchangeable les mots « migration » et « déplacement ». Comme indiqué précédemment, la notion de migration comporte une dimension volontaire alors que la notion de déplacement comporte un élément de coercition.

Ce rapport et la réunion de juin 2009 en question fournissent un exemple récent d'interventions politiques qui favorisent une série d'approches allant au-delà d'un instrument juridique international pour protéger les personnes « déplacées » par les changements environnementaux. La première approche consiste à renforcer la résilience des gens aux impacts du changement climatique, en particulier celle des populations vulnérables. Le but d'une telle approche est de réduire le risque de catastrophes humanitaires et le nombre de personnes forcées à quitter leur lieu de vie. Les deux autres approches soulevées dans le rapport proposent l'intégration des « migrations » dans l'adaptation au changement climatique et le renforcement des mesures juridiques dans certains cas. L'OIM a également joué un rôle central dans ce changement de définition où le déplacement est présenté comme une stratégie d'adaptation au changement climatique ${ }^{58}$.

La préparation à la réunion COP 15 présageait qu'il s'agirait d'un événement majeur dans les négociations internationales sur le changement climatique. Fait largement reconnu aujourd'hui, cette réunion a été un échec quant à son objectif de parvenir à un « accord significatif 59 ", et n'est parvenue qu'à attirer l'attention de la communauté et des médias internationaux. Cet échec est en grande partie lié à l'incapacité des participants à s'entendre sur des réductions significatives des émissions de gaz à effet de serre dans les années à venir. Il semblerait donc que cette réunion ait, par défaut, accepté la « destruction éventuelle 60 » de communautés à travers le monde, en particulier celle des petits États insulaires et des zones de faible altitude. Compte tenu de cela, et à la suite des travaux d'agences onusiennes, d'institutions subsidiaires et de diverses ONG (en collaboration avec l'Organisation des Nations Unies), il aurait été nécessaire de mieux prendre en compte la mobilité humaine comme une des conséquences du changement croissant du climat planétaire.

58. OIM, "Climate change and migrations: Improving methodologies to estimate flows", IOM Migration Research Series (no 33), Genève, OIM, 2008 ; OIM, "Expert seminar: Migration and the environment", IOM International Dialogue on Migration (no 10), Genève, OIM, 2008 ; OIM, "Migration and climate change", IOM Migration Research Series (no 31), Genève, OIM, 2007.

59. Farbotko C., McGregor H. V., "Copenhagen, climate science and the emotional geographies of climate change”, Australian Geographer, 41, 2010, p. 164.

60. Bedford R., Bedford C., "International migration and climate change: A post-Copenhagen perspective on options for Kiribati and Tuvalu", in Burson B. (ed.), Climate Change and Migration: South Pacific Perspectives, Wellington, Institute of Policy Studies, 2010, p. 89. 
Seule une brève mention a été accordée à la question de la mobilité humaine en tant que résultat des crises environnementales, notamment du changement climatique et de l'élévation du niveau de la mer qui en résulte. Suite à un accord au sein du groupe de travail spécial sur l'action concertée à long terme au titre de la Convention, il a été convenu que la COP :

«Invite toutes les parties [...] à entreprendre, notamment : des mesures pour améliorer la compréhension et la coopération, aux niveaux national, régional et international, sur les questions de déplacements liés au changement climatique, de migration et, le cas échéant, de réinstallation planifiée 61 ».

Ici, il est reconnu qu'un lien clair existe entre changement climatique et " déplacement ", ainsi qu'entre changement climatique et « migration » ou "réinstallation planifiée ». Bien qu’à ces trois catégories distinctes correspondent différentes perceptions et « choix » pour les populations vulnérables concernées, il s'agit d'une évolution prometteuse dans le sens où le changement environnemental climatique est reconnu dans l'ordre du jour de la COP comme une cause distincte de la mobilité humaine. Les négociations internationales précédentes sur le changement climatique avaient largement porté leurs efforts (en grande partie, vains) sur l'atténuation et la réduction des émissions de gaz à effet de serre. En dépit de cette reconnaissance tardive, deux (des trois) catégories que la Conférence des Parties a utilisées pour mettre l'accent sur la mobilité humaine due au changement climatique insistent sur la question de la résilience interne. En ce sens, afin de s'adapter, les communautés affectées doivent pouvoir migrer (ce qui implique d'avoir le choix de quitter sa maison) ou doivent pouvoir planifier leur réinstallation (ce qui implique de nouveau d'avoir le choix, les options et les ressources nécessaires). À bien des égards, ces perceptions sont identiques à celles de la CNUED en 1992 présentant les «déplacés» comme devant porter le fardeau du changement, comme des sujets « adaptables».

\section{Conclusion}

Cet article a présenté un aperçu historique de certains documents et rapports dans le but de faire apparâtre les différentes descriptions de la mobilité humaine comme conséquence des changements environnementaux. Cette analyse a été basée sur certains « moments » importants où les organismes des Nations Unies décrivent ceux qui deviennent mobiles suite à de changements environnementaux et produisent certaines catégorisations. À travers ces " moments », différentes catégories ont été créées représentant ces sujets mobiles et leurs comportements, et générant de réponses politiques particu-

61. UNFCCC COP15, Ad Hoc Working Group on Long-term Cooperative Action under the Convention, Copenhagen, UNFCCC, 2009, p. 2. 
lières. Ces descriptions textuelles de personnes déplacées révèlent ainsi comment des processus naturels, des événements et des catastrophes sont perçus et interprétés ${ }^{62}$. Ces interprétations de la « nature » et des changements environnementaux, proposées dans ces textes politiques produits par l'Organisation des Nations Unies et les agences qui lui sont rattachées, influencent la façon dont ces mêmes populations sont perçues et, en retour, comment ces perceptions influencent le champ des politiques publiques.

Les distinctions faites entre les processus environnementaux soudains ou progressifs et les catastrophes ont fortement influencé les différentes représentations de la mobilité humaine liée à ces changements. Les personnes déplacées à la suite de graves perturbations de l'environnement ont tendance à être considérées comme des victimes temporaires et des facteurs aggravant leur propre situation. Bien que les mouvements de personnes lors de changements environnementaux aigus et de court terme puissent faire appel à une intervention d'urgence de la part de l'Organisation des Nations Unies et d'autres institutions, ils n'ont pas donné lieu à l'élaboration d'un cadre de protection institutionnalisé. Ceux qui sont touchés par ces changements ont été perçus comme vivant une situation temporaire.

De même, la façon dont sont interprétés les processus de changements environnementaux lents a déterminé la façon de percevoir ceux qui en sont touchés. La réponse réservée à ces personnes a été considérée comme moins réactive et immédiate, en comparaison avec celle apportée à ceux qui ont été touchés par des catastrophes environnementales brutales. Cela a eu pour effet un blocage dans l'émergence d'actions multilatérales. Dans les différents «moments » du discours politique, ces « déplacés », du fait d'un changement lent de l'environnement, étaient parfois décrits comme les auteurs du changement, ce qui a permis de rejeter sur eux le blâme et la responsabilité de leur situation. À d'autres moments, ces mêmes « déplacés " étaient présentés comme des sujets adaptables, plaçant encore une fois le fardeau de la responsabilité sur les victimes qui devraient seules s'adapter. Enfin, ils ont aussi été parfois perçus comme des victimes réinstallées à l'intérieur de leur pays et, dans ce cas, les gouvernements à l'échelle nationale avaient la charge d'aider et de protéger ces «déplacés». Ces descriptions textuelles ont étouffé toute dynamique potentielle permettant la mise en place d'une politique internationale sur cette question. 